\title{
Quantitative Analytical Method for Eighteen Marker Compounds in Ongyeong-tang, a Traditional Herbal Formula, Using High-Performance Liquid Chromatography for Quality Assessment
}

\author{
Jung-Hoon Kim ${ }^{1,2 \#}$, Hyeun-Kyoo Shin ${ }^{1}$, Chang-Seob Seo ${ }^{1 *}$ \\ 1 : Herbal Medicine Formulation Research Group, Korea Institute of Oriental Medicine, Daejeon, \\ Republic of Korea, 305-811 \\ 2 : Division of Pharmacology, School of Korean Medicine, Pusan National University. Yangsan, \\ Gyeongnam, Republic of Korea, 626-870
}

\begin{abstract}
Objectives : Ongyeong-tang (OGT) is a traditional herbal formula used to cure gynaecological disorders. OGT consists of 12 herbal medicines containing various bioactive components. Therefore, the development of suitable analytical method for the marker compounds is necessary for the quality control of OGT.

Methods : Determination of the 18 marker compounds in OGT preparations was quantitatively performed by high-performance liquid chromatography-photodiode array detection analysis. The marker compounds were separated on a reversed-phase $\mathrm{C}_{18}$ column and the analytical method was successfully validated, which was applied to compare OGT extracts from laboratory preparation and commercial OGT granules.

Results : Limit of detection and limit of quantification values were in the ranges of $0.001-0.016 \mu \mathrm{g} / \mathrm{mL}$ and $0.003-0.047 \mu \mathrm{g} / \mathrm{mL}$, respectively. Precision was $0.03-3.71 \%$ within a day and $0.03-3.81 \%$ over four consecutive days. Recovery of marker compounds ranged from $90.63-108.26 \%$, with relative standard deviation (RSD) values $\left\langle 4.0 \%\right.$. Reproducibility was $\left\langle 2.5 \%\right.$ of the RSD value. The 18 marker compounds were stable within $16 \mathrm{~h}$ at $10{ }^{\circ} \mathrm{C}$, with the RSD value $\langle 3.5 \%$. Quantitative analysis results showed that the quantities of the 18 marker compounds varied among OGT samples. Pearson coefficient evaluation and principal component analysis demonstrated that an OGT water extract produced by a laboratory method clearly differed from commercial OGT granules.

Conclusions : The developed analytical method was simple, precise, and reliable. Therefore, it can be used for the quality assessment of OGT preparations.
\end{abstract}

Key words : Ongyeong-tang, Quantitative analysis, Method development, Pearson coefficient, Principal component analysis

\section{Introduction}

Ongyeong-tang (OGT), a traditional herbal formula for treating gynaecological disorders, consists of 12 herbal medicines, i.e. . Liriope platyphylla F.T.Wang \& Tang, Angelica gigas Nakai, Panax ginseng C.A.Mey., Pinellia ternata (Thunb.) Makino, Paeonia lactiflora Pall., Cnidium officinale Makino., Paeonia suffruticosa Andrews, Equus asinus L., Glycyrrhiza uralensis Fisch., Euodia ruticarpa (A. Juss.) Benth, Cinnamomum cassia Presl, and Zingiber officinale Roscoe. OGT has been traditionally used for the treatment of menstrual diseases such as irregular menstruation, advanced or delayed menstruation, and uterine bleeding ${ }^{1)}$. Clinical studies reported that OGT can treat anovulatory women with high plasma luteinizing hormone levels, improve gonadotropin pulsatile or estradiol

\footnotetext{
*Corresponding author : Chang-Seob Seo. Herbal Medicine Formulation Research Group, Korea Institute of Oriental Medicine, Daejeon, Republic of Korea, 305-811

- Tel : +82-42-868-9361 $\cdot$ E-mail : csseo0914@kiom.re.kr

\#First author : Jung-Hoon Kim. Division of Pharmacology, School of Korean Medicine, Pusan National University. Yangsan, Gyeongnam, Republic of Korea, 626-870

· Tel : +82-51-510-8456 ·E-mail : kmsct@pusan.ac.kr

- Received : 22 June 2015 -Revised : 16 July 2015 · Accepted : 16 July 2015
} 
secretion in the treatment of anovulatory women, or adjunctively treat hormone replacement therapy-resistant menopausal women with depressive ilness ${ }^{2-5)}$. OGT has also shown pharmacological effects such as stimulatory effects on ovarian steroidogenesis and the ovulatory process in the ovary, inhibitory effects on the corticotrophin - releasing factor-induced increase in locomotor activity, and the proliferation of uterine endometrial tissue ${ }^{6-9)}$. OGT is a combination of these 12 herbal medicines with a specific compositional ratio and is generally prescribed as a form of decoction produced by boiling with water. Multiple chemical constituents can be extracted from the compositional herbs; their chemical interaction is thought to contribute their therapeutic effects. Identification of the chemical compounds in OGT and the determination of their quantities may be a fundamental step towards further pharmacological or clinical experiments. However, the quantification of OGT based on a single compound, or several chemical compounds is challenging, because a few compounds cannot reflect all the chemical characteristics of OGT. Therefore, the simultaneous determination of various chemical compounds with different structure is necessary for understanding the full chemical spectrum of OGT. Thus, the quality assessment of OGT preparations can be performed using quantitative analysis of multiple components as marker compounds.

High-performance liquid chromatography combined with photodiode array detection (HPLC-PDA) has been widely used for the chemical analysis of herbal formulae; it is a simple, precise, and rapid technique for the detection of multiple chemical compounds ${ }^{10-12)}$. Thus, HPLC-PDA methods are used for the quality control of herbal preparations ${ }^{13-15)}$. Various bioactive marker compounds were reported in the compositional herbs of OGT: gallic acid, oxypaeoniflorin, (+)-catechin, paeoniflorin, 1,2,3,4,6-pentagalloylglucose, benzoylpaeoniflorin, and paeonol from $P$. lactiflora and $P$. suffruticosa; chlorogenic acid, caffeic acid, and ferulic acid from $C$. officinale; liquiritin, ononin, and glycyrrhizin from $G$. uralensis; nodakenin from $A$. gigas; coumarin, cinnamic acid, and cinnamaldehyde from $C$. cassia; and 6-gingerol from $Z$. officinale ${ }^{16-25)}$. Some of these bioactive compounds were also used as the marker compounds for HPLC analysis of herbal medicines ${ }^{26,27)}$. Therefore, the above 18 chemical compounds can be used as the marker compounds for the quantitative analysis and quality assessment of OGT preparations.

In the present study, we developed analytical methods for the 18 marker compounds in an OGT water extract (OGTWE) by a validated method using HPLC-PDA. The amounts of marker compounds were quantified. An OGTWE and commercial granules were compared for the quality assessment of OGT preparations.

\section{Materials and methods}

\section{Chemicals and reagents}

HPLC-grade methanol, acetonitrile, and water were purchased from J.T. Baker Inc. (Phillipsburg, NJ, USA). Formic acid was purchased from Fluka (Seelze, Germany). Trifluoroacetic acid was purchased from Alfa Aesar (Ward Hill, MA, USA). Gallic acid (1), (+)-catechin (3), ferulic acid (7), coumarin (11), and cinnamic acid (13) were purchased from Sigma-Aldrich (St Louis, MO, USA). Oxypaeoniflorin (2), nodakenin (9), 1,2,3,4,6-pentagalloylglucose (10), ononin (12), and benzoylpaeoniflorin (15) were supplied by Chengdu Biopurify Phytochemicals (Chengdu, China). Chlorogenic acid (4) and caffeic acid (5) were purchased from Acros Organics (Morris Plains, NJ, USA). Paeoniflorin (6), cinnamaldehyde (14), and paeonol (16), 6-gingerol (17), and glycyrrhizin (18) were obtained from Wako Pure Chemical Industries (Osaka, Japan). Liquiritin (8) was supplied by NPC Biotechnology (Geumsan, Korea). The chemical structures of the standard compounds are shown in Fig. 1. The purity of all reagents and marker compounds was $\geq 98 \%$.

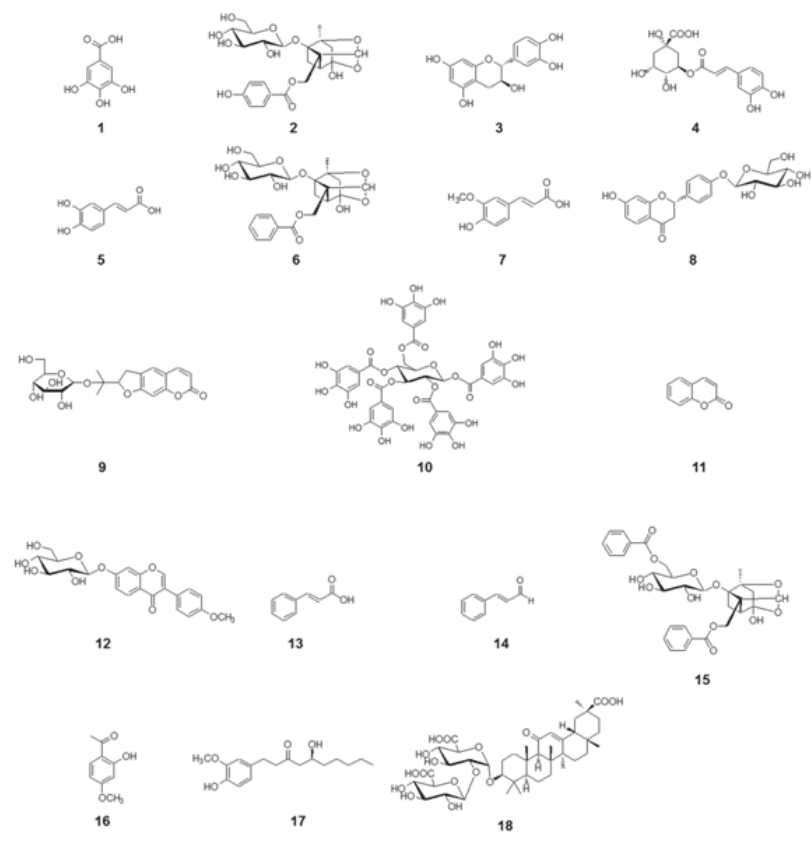

Fig. 1. Chemical structures of the 18 standard compounds in Ongyeong-tang (OGT). (1) Gallic acid, (2) oxypaeoniflorin, (3) (+)-catechin, (4) chlorogenic acid, (5) caffeic acid, (6) paeoniflorin, (7) ferulic acid, (8) liquiritin, (9) nodakenin, (10) 1,2,3,4,6-pentagalloylglucose, (11) coumarin, (12) ononin, (13) cinnamic acid, (14) cinnamaldehyde, (15) benzoylpaeoniflorin, (16) paeonol, (17) 6-gingerol, and (18) glycyrrhizin.

Compositional herbal medicines purchased from the herbal medicine company, Kwangmyungdang Medicinal Herbs (Ulsan, Korea), and from the commercial samples are listed in Table 1. A voucher specimen (2014-KE41-1 
-12) was deposited in the Herbal Medicine Formulation Research Group of the Korea Institute of Oriental Medicine.

Table 1. Composition of Ongyeong-tang water extract (OGTWE), OGT01, and OGT02

\begin{tabular}{|c|c|c|c|c|}
\hline \multirow{2}{*}{ Herbal medicine } & \multicolumn{2}{|l|}{ OGTWE } & \multirow{2}{*}{$\begin{array}{l}\text { OGT01 } \\
\text { (g) }\end{array}$} & \multirow{2}{*}{$\begin{array}{l}\text { OGT02 } \\
(\mathrm{g})\end{array}$} \\
\hline & Original region & $\underset{(\mathrm{g})}{\text { Amount }}$ & & \\
\hline Liriope platyphylla F.T.Wang \& Tang & Milyaong, Gyeongnam, Korea & 7.50 & 3.33 & 3.33 \\
\hline Angelica gigas Nakai & Bonghwa, Gyeongbuk, Korea & 5.63 & 1.00 & 1.00 \\
\hline Panax ginseng C.A.Mey. & Yeongju, Gyeongbuk, Korea & 3.75 & 0.67 & 0.67 \\
\hline Pinellia ternata (Thunb.) Makino & China & 3.75 & 1.67 & 1.67 \\
\hline Paeonia lactiflora Pall. & Euiseong, Gyeongbuk, Korea & 3.75 & 0.67 & 0.67 \\
\hline Cnidium officinale Makino. & $\begin{array}{l}\text { Yeongyang, Gyeongbuk, } \\
\text { Korea }\end{array}$ & 3.75 & 0.67 & 0.67 \\
\hline Paeonia suffruticosa Andrews & Jecheon, Chungbuk, Korea & 3.75 & 0.67 & 0.67 \\
\hline Equus asinus $\mathrm{L}$. & China & 2.81 & 0.67 & 0.67 \\
\hline Glycyrrhiza uralensis Fisch. & China & 2.81 & 0.67 & 0.67 \\
\hline Euodia ruticarpa (A. Juss.) Benth & China & 1.88 & 1.00 & 1.00 \\
\hline Cinnamomum cassia Presl & Vietnam & 1.88 & 0.67 & 0.67 \\
\hline Zingiber officinale Roscoe & Ulsan, Gyeongbuk, Korea & 3.75 & 0.33 & 0.33 \\
\hline Sum & - & 45.01 & 12.02 & 12.02 \\
\hline
\end{tabular}

\section{Sample preparation of OGT WE and} commercial granules

A mixture of crude herbal drugs consisting of OGT was extracted with a 10-fold volume of distilled water $(\mathrm{w} / \mathrm{v})$ at $100{ }^{\circ} \mathrm{C}$ for $2 \mathrm{~h}$ under pressure $\left(1 \mathrm{kgf} / \mathrm{cm}^{2}\right)$ using an electric extractor (COSMOS-660; Kyungseo Machine, Incheon, Korea). The extracted decoction was filtered through a standard sieve (no. 270, $53 \mu \mathrm{m}$; Chunggyesangongsa, Seoul, Korea) and then freeze-dried to obtain an OGTWE powder.

Accurately weighed OGTWE $(100 \mathrm{mg})$ and commercial OGT granules $(300 \mathrm{mg})$ were dissolved in $10 \mathrm{~mL}$ distilled water, and the solutions were filtered through a $0.2 \mu \mathrm{m}$ syringe filter (SmartPor ${ }^{\circledR}$; Woongki Science, Seoul, Korea) prior to injection into the HPLC apparatus.

\section{Preparation of standard solutions of marker}

\section{compounds}

The stock solutions were prepared by dissolving accurately weighed standard compounds in methanol at concentrations of $1000 \mathrm{\mu g} / \mathrm{mL}$. Working solutions were produced by diluting the stock solutions containing standard compounds. Diluted working solutions were used to construct calibration curves.

\section{Chromatographic conditions}

The HPLC-PDA system comprised a LC-20A (Shimadzu, Kyoto, Japan) equipped with a solvent delivery unit, autosampler, column oven, degasser, and PDA. The acquired data were processed using LabSolutions software (v. 5.3; Shimadzu). The 18 marker compounds were separated on a Gemini $\mathrm{C}_{18}$ column $(4.6 \times 250 \mathrm{~mm}$, $5 \mathrm{\mu m}$; Phenomenex, Torrance, CA, USA). The column oven was maintained at $40{ }^{\circ} \mathrm{C}$. The mobile phase consisted of water containing $0.1 \%$ formic acid (A) and acetonitrile (B), with gradient elution (see Table 2 ). The flow rate was $1.0 \mathrm{~mL} / \mathrm{min}$ and the injection volume was set to $10 \mu \mathrm{L}$. The detection wavelengths were optimized according to the maximum absorption wavelengths of the standard compounds.

Table 2. Mobile phase gradient conditions (A:B)

\begin{tabular}{ccc}
\hline Time $(\min )$ & A (\%) & B (\%) \\
\hline \hline 0 & 92 & 8 \\
10 & 83 & 17 \\
15 & 83 & 17 \\
25 & 80 & 20 \\
28 & 80 & 20 \\
32 & 72 & 28 \\
35 & 72 & 28 \\
45 & 44 & 56 \\
47 & 44 & 56 \\
55 & 23 & 77 \\
57 & 23 & 77 \\
65 & 92 & 8 \\
72 & 92 & 8 \\
\hline
\end{tabular}

A, $0.1 \%$ formic acid in water; B, acetonitrile.

\section{Method validation}

\section{1) Linearity}

Accurately weighed standard compounds were dissolved in methanol to prepare stock solutions in concentrations of $1000 \mathrm{\mu g} / \mathrm{mL}$. Stock solutions of the 18 marker compounds were diluted to construct calibration curves. The diluted concentrations of marker compounds were plotted against the peak area on the calibration curves and the linearity was determined using correlation coefficient.

\section{2) The limit of detection (LOD) and limit of quantification} (LOQ)

LOD and LOQ were determined as follows: $\mathrm{LOD}=$ $3.3 \times \mathrm{SD} / \mathrm{S}$ and $\mathrm{LOQ}=10 \times \mathrm{SD} / \mathrm{S}$, where $\mathrm{SD}$ is the standard deviation of the response and $\mathrm{S}$ is the slope of the calibration curve.

\section{3) Precision}

The precision was evaluated by four analyses in one day (intra-day precision) and by analysis over four consecutive days (inter-day precision. Precision was calculated by analysing sample extracts containing 
low, medium, and high concentrations of marker compounds. The precision value was represented as the relative standard deviation (RSD) [(standard deviation / mean) $\times 100]$.

\section{4) Recovery}

The recovery test was performed to evaluate the accuracy of the method used. The three known amounts of marker compounds (low, medium, and high) were added to the samples and the recovery was calculated as follows: recovery $(\%)=[($ detected concentration - initial concentration) / spiked concentration) $\times 100]$.

\section{5) Reproducibility}

The reproducibility was determined by calculating the RSD values of the retention times and the absolute areas of marker compounds $(n=6)$.

\section{6) Stability}

The stability test was conducted by analysing the extracted solution after storage under refrigeration at $10{ }^{\circ} \mathrm{C}$ for $4,8,12$, and $16 \mathrm{~h}$

\section{Statistical analysis}

One-way analysis of variance (ANOVA) using the Tukey test, Pearson correlation efficient, and the principal component analysis (PCA) were performed for multiple group comparisons using open-source software $\mathrm{R}$ (v. 3.0.2). Differences were considered significant at $P<0.05$, $P<0.01$, or $P<0.001$.

\section{Results}

\section{Optimization of chromatographic conditions}

The mobile phase consisted of water (A) and acetonitrile (B), and a modifier. Two different modifiers, such as $0.1 \%$ trifluoroacetic acid and $0.1 \%$ formic acid, were compared for the optimal separation of the 18 marker compounds. Better resolution and peak shapes between marker compounds were obtained when $0.1 \%$ formic acid was used as modifier. Various ratios of the components of the mobile phase (A:B) were tested using gradient elution for optimal separation of marker compounds. Selected gradient elution conditions used are listed in TableII. The optimal UV wavelength for each marker compound was tested in the range 190-400 nm: paeoniflorin and benzoylpaeoniflorin at $230 \mathrm{~nm}$; ononin and glycyrrhizin at $250 \mathrm{~nm}$; oxypaeoniflorin at $255 \mathrm{~nm}$; gallic acid at $270 \mathrm{~nm}$; (+)-catechin, liquiritin, coumarin, cinnamic acid, and paeonol at $275 \mathrm{~nm} ; 1,2,3,4,6$-pentagalloylglucose and 6-gingerol at $280 \mathrm{~nm}$; cinnamaldehyde at $290 \mathrm{~nm}$; chlorogenic acid, caffeic acid, and ferulic acid at 325 $\mathrm{nm}$; and nodakenin at $335 \mathrm{~nm}$. The 18 marker compounds were reasonably well separated for quantitative analysis on a chromatogram without severe overlapping or interception of adjacent peaks(Fig. 2).
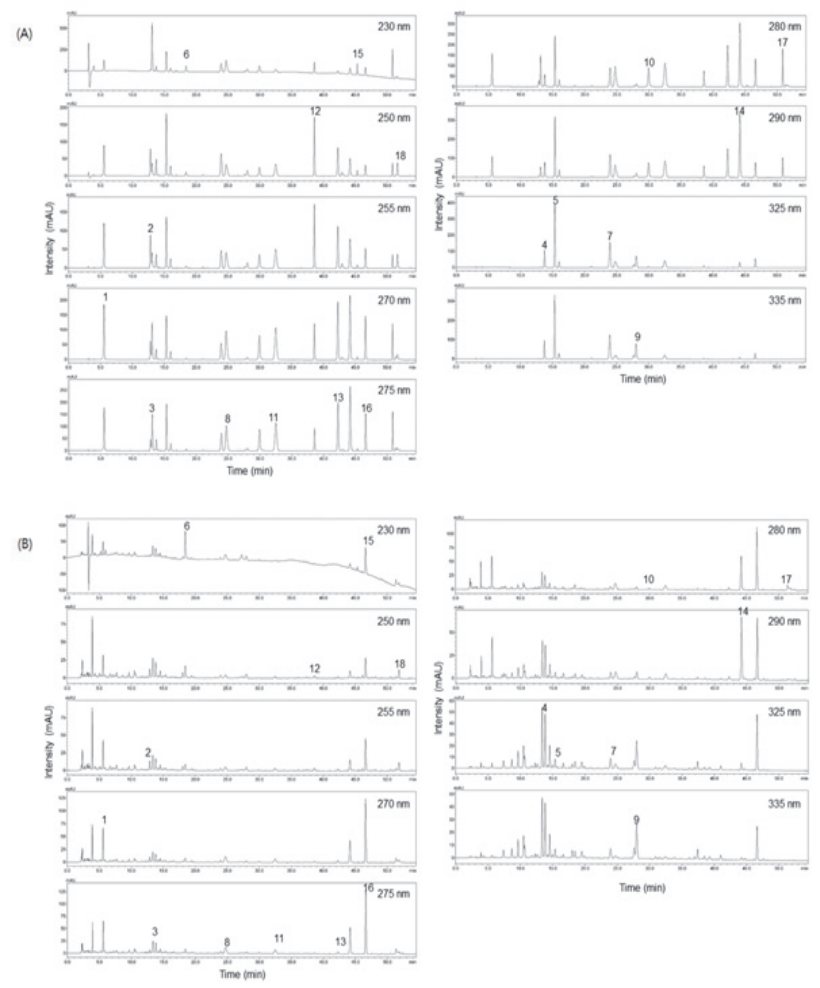

Fig. 2. Chromatograms of the 18 standard compounds (A) and OGT water extract (B). (1) Gallic acid, (2) oxypaeoniflorin, (3) (+)-catechin, (4) chlorogenic acid, (5) caffeic acid, (6) paeoniflorin, (7) ferulic acid, (8) liquiritin, (9) nodakenin, (10) 1,2,3,4,6-pentagalloylglucose, (11) coumarin, (12) ononin, (13) cinnamic acid, (14) cinnamaldehyde, (15) benzoylpaeoniflorin, (16) paeonol, (17) 6-gingerol, and (18) glycyrrhizin.

\section{Method validation}

\section{1) System suitability}

The system suitability was measured in terms of the capacity factor (k), relative retention (a), resolution (Rs), theoretical plate number $(\mathrm{N})$, and symmetry factor $(\mathrm{S})$ of the 18 marker compounds. The capacity factor ranged from 0.81 to 15.60 . The relative retention was $1<\mathrm{a}<$ 4 and the resolutions of marker compounds were $>$ 1.2, which indicate that the marker peaks were not severely overlapped by adjacent peaks and interference from other components. The number of theoretical plates was $>8944$. The symmetry factor was $0.9<\mathrm{S}<$ 1.6, which demonstrated that peak fronting or tailing was not found(Table 3). 
Table 3. System suitability data

\begin{tabular}{lccccc}
\hline \multicolumn{1}{c}{ Compound } & $\mathrm{k}$ & $\alpha$ & $\mathrm{Rs}$ & $\mathrm{N}$ & $\mathrm{S}$ \\
\hline \hline Gallic acid & 0.81 & 3.94 & 33.73 & 8944.47 & 0.98 \\
Oxypaeoniflorin & 3.18 & 1.03 & 1.22 & 66669.90 & 1.07 \\
(+)-Catechin & 3.26 & 1.03 & 1.22 & 57929.90 & 1.04 \\
Chlorogenic acid & 3.48 & 1.06 & 3.10 & 75069.98 & 1.11 \\
Caffeic acid & 3.99 & 1.15 & 7.64 & 79454.31 & 1.07 \\
Paeoniflorin & 4.94 & 1.25 & 12.25 & 65062.78 & 1.52 \\
Ferulic acid & 6.80 & 1.04 & 1.82 & 57503.58 & 1.02 \\
Liquiritin & 7.05 & 1.04 & 1.82 & 47476.51 & 1.29 \\
Nodakenin & 8.14 & 1.15 & 8.18 & 96048.90 & 0.99 \\
1,2,3,4,6-Pentagalloylglucose & 8.75 & 1.08 & 5.11 & 98953.30 & 1.13 \\
Coumarin & 9.59 & 1.10 & 5.35 & 50320.04 & 0.99 \\
Ononin & 11.41 & 1.21 & 15.28 & 503128.80 & 1.10 \\
Cinnamic acid & 12.77 & 1.10 & 13.58 & 268351.87 & 1.02 \\
Cinnamaldehyde & 13.39 & 1.05 & 5.89 & 293251.85 & 1.04 \\
Benzoylpaeoniflorin & 13.60 & 1.03 & 4.66 & 1234168.88 & 1.41 \\
Paeonol & 14.19 & 1.03 & 6.52 & 631799.59 & 1.07 \\
6-Gingerol & 15.57 & 1.02 & 3.65 & 1553984.63 & 1.10 \\
Glycyrrhizin & 15.60 & 1.02 & 3.65 & 655683.08 & 1.09 \\
\hline
\end{tabular}

$\mathrm{k}$, capacity factor; a, relative retention; Rs, resolution; N, theoretical plate; S, symmetry factor.

\section{2) Linear regression, LOD, and LOQ}

The linearity was represented as the correlation coefficient $\left(r^{2}\right)$ of compounds: it ranged from 0.9998 to 1.0000. The LOD and LOQ values were $0.000-0.016$ $\mu \mathrm{g} / \mathrm{mL}$ and $0.001-0.047 \mu \mathrm{g} / \mathrm{mL}$, respectively(Table 4).

Table 4. Linear equation, correlation coefficients $\left(r^{2}\right)$, LOD ,and LOQ fo rthe marker compounds in OGT

\begin{tabular}{lcccccc}
\hline \multicolumn{1}{c}{ Compound } & $\begin{array}{c}\text { Detection } \\
\text { wavelength } \\
(\mathrm{nm})\end{array}$ & Linear equation & $r^{2}$ & $\begin{array}{c}\text { Linear } \\
\text { range } \\
(\mu \mathrm{\mu g} / \mathrm{mL})\end{array}$ & $\begin{array}{c}\text { LOD } \\
(\mu \mathrm{g} / \mathrm{mL})\end{array}$ & $\begin{array}{c}\mathrm{LOQ} \\
(\mathrm{\mu g} / \mathrm{mL})\end{array}$ \\
\hline \hline Gallic acid & 270 & $\mathrm{y}=37527 \mathrm{x}-9073.9$ & 0.9999 & $0.94-60$ & 0.002 & 0.007 \\
Oxypaeoniflorin & 255 & $\mathrm{y}=13639 \mathrm{x}-1395.9$ & 1.0000 & $0.63-40$ & 0.010 & 0.030 \\
$(+)$-Catechin & 275 & $\mathrm{y}=6309.1 \mathrm{x}-102.97$ & 0.9999 & $0.08-5$ & 0.016 & 0.047 \\
Chlorogenic acid & 325 & $\mathrm{y}=37099 \mathrm{x}-8987.9$ & 0.9999 & $0.94-60$ & 0.002 & 0.005 \\
Caffeic acid & 325 & $\mathrm{y}=50288 \mathrm{x}-1834.7$ & 0.9999 & $0.16-10$ & 0.001 & 0.004 \\
Paeoniflorin & 230 & $\mathrm{y}=13648 \mathrm{x}-5626.6$ & 1.0000 & $3.91-250$ & 0.005 & 0.015 \\
Ferulic acid & 325 & $\mathrm{y}=44083 \mathrm{x}-4028.1$ & 1.0000 & $0.47-30$ & 0.001 & 0.004 \\
Liquiritin & 275 & $\mathrm{y}=20603 \mathrm{x}-3948.9$ & 1.0000 & $1.17-75$ & 0.005 & 0.014 \\
Nodakenin & 335 & $\mathrm{y}=31107 \mathrm{x}-8810.6$ & 1.0000 & $1.56-100$ & 0.003 & 0.009 \\
1,2,3,4,6-Pentagalloylglucose & 280 & $\mathrm{y}=24679 \mathrm{x}-4035.9$ & 0.9998 & $0.31-20$ & 0.004 & 0.011 \\
Coumarin & 275 & $\mathrm{y}=53552 \mathrm{x}-8072.9$ & 1.0000 & $0.78-50$ & 0.002 & 0.006 \\
Ononin & 250 & $\mathrm{y}=31487 \mathrm{x}-661.44$ & 1.0000 & $0.12-7.5$ & 0.002 & 0.007 \\
Cinnamic acid & 275 & $\mathrm{y}=64369 \mathrm{x}-1459.6$ & 1.0000 & $0.12-7.5$ & 0.002 & 0.005 \\
Cinnamaldehyde & 290 & $\mathrm{y}=140371 \mathrm{x}-9931.9$ & 1.0000 & $0.49-31.5$ & 0.000 & 0.001 \\
Benzoylpaeoniflorin & 230 & $\mathrm{y}=24942 \mathrm{x}-4605.7$ & 0.9999 & $0.39-25$ & 0.003 & 0.008 \\
Paeonol & 275 & $\mathrm{y}=54045 \mathrm{x}-1984.1$ & 1.0000 & $1.95-125$ & 0.002 & 0.006 \\
6-Gingerol & 280 & $\mathrm{y}=6131.2 \mathrm{x}+31.083$ & 0.9998 & $0.08-2.5$ & 0.014 & 0.044 \\
Glycyrrhizin & 250 & $\mathrm{y}=7812.5 \mathrm{x}-246.21$ & 1.0000 & $1.56-100$ & 0.009 & 0.028 \\
\hline
\end{tabular}

LOD, limit of detection; LOQ, limit of quantification; y, peak area $(\mathrm{mAU}) ; \mathrm{x}$, concentration of compound $(\mu \mathrm{g} / \mathrm{mL})$.

\section{3) Precision, recovery, reproducibility, and stability}

The intra- and inter-day precisions were represented as RSD values $<4.0 \%$ in three concentration levels (Table 5). The recoveries of the 18 marker compounds were in the range $90.63-107.57 \%$, with RSD values < $4.0 \%$, at three different spiked concentrations(Table 6).
The reproducibility of the marker compounds was also represented as RSD values; $<0.2 \%$ for the retention time and $\langle 2.5 \%$ for absolute area. The stability did not exceed $3.5 \%$ of the RSD value (Table 7). These results indicate that the analytical method that was developed was precise, reproducible, and stable for the analysis of the 18 marker compounds in OGT samples.

Table 5. Intra- and inter-day precision of the 18 marker compounds in OGT

\begin{tabular}{|c|c|c|c|c|c|c|c|}
\hline \multirow[b]{2}{*}{ Compound } & \multirow[b]{2}{*}{$\begin{array}{l}\text { Spiked } \\
\text { conc. } \\
(\text { (ug/mL) }\end{array}$} & \multicolumn{3}{|c|}{ Intra-day $(n=4)$} & \multicolumn{3}{|c|}{ Inter-day $(n=4)$} \\
\hline & & $\begin{array}{c}\text { Detected } \\
\text { conc. } \\
(\mu \mathrm{g} / \mathrm{mL})\end{array}$ & $\begin{array}{l}\mathrm{RSD} \\
(\%)\end{array}$ & $\begin{array}{c}\text { Accuracy } \\
(\%)\end{array}$ & $\begin{array}{c}\text { Detected } \\
\text { conc. } \\
(\mu \mathrm{g} / \mathrm{mL})\end{array}$ & $\begin{array}{l}\mathrm{RSD} \\
(\%)\end{array}$ & $\begin{array}{c}\text { Accuracy } \\
(\%)\end{array}$ \\
\hline \multirow{3}{*}{ Gallic acid } & 2.50 & 2.46 & 0.89 & 97.40 & 2.48 & 1.15 & 98.16 \\
\hline & 5.00 & 4.90 & 0.84 & 98.15 & 4.90 & 0.84 & 97.74 \\
\hline & 10.00 & 10.06 & 0.20 & 100.63 & 10.06 & 0.20 & 100.68 \\
\hline \multirow{3}{*}{ Oxypaeoniflorin } & 2.00 & 1.92 & 1.75 & 97.86 & 1.92 & 1.89 & 97.86 \\
\hline & 4.00 & 4.07 & 0.43 & 102.11 & 4.06 & 0.45 & 102.11 \\
\hline & 8.00 & 7.99 & 0.18 & 99.60 & 7.99 & 0.20 & 99.60 \\
\hline \multirow{3}{*}{$(+)$-Catechin } & 0.50 & 0.50 & 1.64 & 100.99 & 0.50 & 1.05 & 100.99 \\
\hline & 1.00 & 0.97 & 1.56 & 94.85 & 0.96 & 1.29 & 94.85 \\
\hline & 2.00 & 2.02 & 0.28 & 101.23 & 2.02 & 0.25 & 101.23 \\
\hline \multirow{3}{*}{ Chlorogenic acid } & 2.50 & 2.49 & 0.55 & 99.57 & 2.47 & 1.00 & 99.57 \\
\hline & 5.00 & 4.87 & 0.48 & 97.68 & 4.86 & 0.72 & 97.68 \\
\hline & 10.00 & 10.07 & 0.10 & 100.61 & 10.08 & 0.23 & 100.61 \\
\hline \multirow{3}{*}{ Caffeic acid } & 1.00 & 0.98 & 0.65 & 98.02 & 0.99 & 0.75 & 98.02 \\
\hline & 2.00 & 1.90 & 1.34 & 96.24 & 1.89 & 2.21 & 96.24 \\
\hline & 4.00 & 4.05 & 0.32 & 101.06 & 4.06 & 0.47 & 101.06 \\
\hline \multirow{3}{*}{ Paeoniflorin } & 15.00 & 15.05 & 2.27 & 100.99 & 14.87 & 1.35 & 98.36 \\
\hline & 30.00 & 29.89 & 1.09 & 100.31 & 30.59 & 3.81 & 107.70 \\
\hline & 60.00 & 60.04 & 0.25 & 99.86 & 59.74 & 0.96 & 98.17 \\
\hline \multirow{3}{*}{ Ferulic acid } & 1.00 & 1.07 & 0.71 & 107.57 & 1.07 & 1.63 & 109.72 \\
\hline & 2.00 & 2.08 & 0.83 & 103.11 & 2.06 & 2.36 & 99.41 \\
\hline & 4.00 & 3.94 & 0.20 & 98.75 & 3.95 & 0.51 & 99.54 \\
\hline \multirow{3}{*}{ Liquiritin } & 3.00 & 3.00 & 1.47 & 100.40 & 3.00 & 1.78 & 100.40 \\
\hline & 6.00 & 5.97 & 0.87 & 98.45 & 5.98 & 0.86 & 98.45 \\
\hline & 12.00 & 12.02 & 0.26 & 100.36 & 12.01 & 0.23 & 100.36 \\
\hline \multirow{3}{*}{ Nodakenin } & 4.00 & 3.79 & 0.82 & 95.36 & 3.77 & 1.30 & 95.36 \\
\hline & 8.00 & 7.89 & 0.22 & 98.39 & 7.89 & 0.18 & 98.39 \\
\hline & 16.00 & 16.11 & 0.07 & 100.69 & 16.11 & 0.08 & 100.69 \\
\hline \multirow{3}{*}{ 1,2,3,4,6-Pentagalloylglucose } & 0.50 & 0.51 & 1.97 & 99.22 & 0.50 & 2.00 & 99.22 \\
\hline & 1.00 & 1.05 & 1.21 & 105.33 & 1.06 & 1.69 & 105.33 \\
\hline & 2.00 & 1.97 & 0.23 & 98.72 & 1.97 & 0.36 & 98.72 \\
\hline \multirow{3}{*}{ Coumarin } & 1.00 & 1.07 & 0.64 & 107.47 & 1.07 & 0.73 & 107.47 \\
\hline & 2.00 & 2.10 & 0.28 & 105.23 & 2.11 & 0.29 & 105.23 \\
\hline & 4.00 & 3.93 & 0.09 & 98.23 & 3.93 & 0.04 & 98.23 \\
\hline \multirow{3}{*}{ Ononin } & 0.50 & 0.51 & 0.40 & 102.08 & 0.52 & 1.86 & 102.08 \\
\hline & 1.00 & 0.99 & 0.16 & 99.49 & 0.99 & 0.35 & 99.49 \\
\hline & 2.00 & 2.00 & 0.03 & 100.00 & 2.00 & 0.05 & 100.00 \\
\hline \multirow{3}{*}{ Cinnamic acid } & 0.50 & 0.49 & 1.86 & 98.84 & 0.50 & 2.09 & 98.84 \\
\hline & 1.00 & 1.01 & 0.65 & 101.77 & 1.01 & 1.02 & 101.77 \\
\hline & 2.00 & 2.00 & 0.15 & 99.63 & 2.00 & 0.19 & 99.63 \\
\hline & 1.05 & 0.95 & 1.08 & 89.89 & 0.95 & 1.31 & 89.89 \\
\hline Cinnamaldehyde & 2.10 & 2.19 & 0.50 & 104.44 & 2.19 & 0.35 & 104.44 \\
\hline & 4.20 & 4.18 & 0.09 & 99.52 & 4.18 & 0.07 & 99.52 \\
\hline & 1.00 & 0.95 & 2.10 & 94.37 & 0.96 & 1.37 & 94.37 \\
\hline Benzoylpaeoniflorin & 2.00 & 2.10 & 2.00 & 102.71 & 2.08 & 3.17 & 102.71 \\
\hline & 4.00 & 3.96 & 0.63 & 99.68 & 3.97 & 0.87 & 99.68 \\
\hline & 4.00 & 3.95 & 1.02 & 98.12 & 3.94 & 1.51 & 98.12 \\
\hline Paeonol & 8.00 & 7.68 & 0.31 & 96.32 & 7.70 & 0.32 & 96.32 \\
\hline & 16.00 & 16.17 & 0.11 & 101.04 & 16.16 & 0.03 & 101.04 \\
\hline & 0.40 & 0.41 & 1.17 & 102.42 & 0.41 & 1.11 & 102.77 \\
\hline 6-Gingerol & 0.80 & 0.79 & 3.71 & 96.17 & 0.81 & 3.80 & 97.99 \\
\hline & 1.60 & 1.60 & 0.91 & 100.81 & 1.59 & 0.96 & 100.33 \\
\hline & 4.00 & 3.94 & 2.05 & 98.07 & 3.93 & 2.46 & 98.07 \\
\hline Glycyrrhizin & 8.00 & 7.79 & 0.16 & 97.54 & 7.77 & 0.41 & 97.54 \\
\hline & 16.00 & 16.12 & 0.12 & 100.74 & 16.13 & 0.22 & 100.74 \\
\hline
\end{tabular}

Conc., concentration; SD, standard deviation; RSD, relative standard deviation $(\%)=(\mathrm{SD} /$ mean $) \times 100$ 
Table 6. Recovery and repeatability of the 18 marker compounds in OGT $(n=5)$

\begin{tabular}{|c|c|c|c|c|c|}
\hline Compound & $\begin{array}{c}\text { Initial conc. } \\
(\mu \mathrm{g} / \mathrm{mL})\end{array}$ & $\begin{array}{c}\text { Spiked conc. } \\
(\mu \mathrm{g} / \mathrm{mL})\end{array}$ & $\begin{array}{c}\text { Detected conc. } \\
(\mu \mathrm{g} / \mathrm{mL})\end{array}$ & $\begin{array}{c}\text { Recovery } \\
(\%)\end{array}$ & $\begin{array}{l}\text { RSD } \\
(\%)\end{array}$ \\
\hline & & 2.50 & 12.25 & 99.17 & 1.77 \\
\hline \multirow[t]{3}{*}{ Gallic acid } & 9.77 & 5.00 & 14.75 & 99.69 & 0.91 \\
\hline & & 10.00 & 20.02 & 102.52 & 1.06 \\
\hline & & 2.00 & 8.19 & 93.95 & 2.84 \\
\hline \multirow[t]{3}{*}{ Oxypaeoniflorin } & 6.32 & 4.00 & 10.30 & 99.64 & 1.10 \\
\hline & & 8.00 & 14.15 & 97.92 & 0.37 \\
\hline & & 0.50 & 1.44 & 99.11 & 2.67 \\
\hline \multirow[t]{3}{*}{$(+)$-Catechin } & 0.94 & 1.00 & 1.94 & 99.69 & 1.96 \\
\hline & & 2.00 & 3.04 & 104.72 & 1.25 \\
\hline & & 2.50 & 12.21 & 94.79 & 1.29 \\
\hline \multirow[t]{3}{*}{ Chlorogenic acid } & 9.84 & 5.00 & 14.54 & 94.04 & 1.61 \\
\hline & & 10.00 & 19.63 & 97.92 & 1.11 \\
\hline & & 1.00 & 2.16 & 98.88 & 1.10 \\
\hline \multirow[t]{3}{*}{ Caffeic acid } & 1.17 & 2.00 & 3.17 & 99.97 & 0.42 \\
\hline & & 4.00 & 5.48 & 107.57 & 0.95 \\
\hline & & 15.00 & 82.57 & 99.82 & 1.85 \\
\hline \multirow[t]{3}{*}{ Paeoniflorin } & 67.60 & 30.00 & 97.81 & 100.70 & 1.36 \\
\hline & & 60.00 & 128.08 & 100.80 & 1.52 \\
\hline & & 1.00 & 4.03 & 108.26 & 1.06 \\
\hline \multirow[t]{3}{*}{ Ferulic acid } & 2.95 & 2.00 & 4.98 & 101.42 & 1.63 \\
\hline & & 4.00 & 6.72 & 94.20 & 0.55 \\
\hline & & 3.00 & 15.31 & 96.69 & 1.81 \\
\hline \multirow[t]{3}{*}{ Liquiritin } & 12.41 & 6.00 & 18.14 & 95.49 & 1.44 \\
\hline & & 12.00 & 23.92 & 95.97 & 2.30 \\
\hline & & 4.00 & 19.70 & 93.38 & 2.09 \\
\hline \multirow[t]{3}{*}{ Nodakenin } & 15.97 & 8.00 & 23.95 & 99.75 & 1.18 \\
\hline & & 16.00 & 32.51 & 103.35 & 0.88 \\
\hline & & 0.50 & 2.47 & 104.49 & 2.62 \\
\hline \multirow[t]{3}{*}{ 1,2,3,4,6-Pentagalloylglucose } & 1.94 & 1.00 & 2.99 & 104.30 & 0.77 \\
\hline & & 2.00 & 3.89 & 97.35 & 0.71 \\
\hline & & 1.00 & 4.66 & 105.64 & 0.49 \\
\hline \multirow[t]{3}{*}{ Coumarin } & 3.60 & 2.00 & 5.59 & 99.31 & 0.61 \\
\hline & & 4.00 & 7.23 & 90.63 & 0.58 \\
\hline & & 0.50 & 1.46 & 105.33 & 0.87 \\
\hline \multirow[t]{3}{*}{ Ononin } & 0.94 & 1.00 & 1.95 & 101.35 & 0.20 \\
\hline & & 2.00 & 2.97 & 101.56 & 0.20 \\
\hline & & 0.50 & 1.43 & 101.13 & 2.42 \\
\hline \multirow[t]{3}{*}{ Cinnamic acid } & 0.92 & 1.00 & 1.97 & 105.07 & 1.35 \\
\hline & & 2.00 & 2.99 & 103.57 & 0.41 \\
\hline & & 1.05 & 7.52 & 91.58 & 1.24 \\
\hline \multirow[t]{3}{*}{ Cinnamaldehyde } & 6.56 & 2.10 & 8.81 & 107.03 & 0.67 \\
\hline & & 4.20 & 10.87 & 102.59 & 0.44 \\
\hline & & 1.00 & 3.70 & 94.89 & 0.62 \\
\hline \multirow[t]{3}{*}{ Benzoylpaeoniflorin } & 2.75 & 2.00 & 4.80 & 102.63 & 1.67 \\
\hline & & 4.00 & 6.73 & 99.66 & 2.65 \\
\hline & & 4.00 & 27.54 & 96.55 & 0.94 \\
\hline \multirow[t]{3}{*}{ Paeonol } & 23.68 & 8.00 & 31.35 & 95.89 & 0.90 \\
\hline & & 16.00 & 40.00 & 102.01 & 0.73 \\
\hline & & 0.40 & 0.51 & 96.66 & 2.47 \\
\hline \multirow[t]{3}{*}{ 6-Gingerol } & 0.13 & 0.80 & 0.87 & 93.10 & 1.56 \\
\hline & & 1.60 & 1.67 & 96.42 & 0.36 \\
\hline & & 4.00 & 17.43 & 100.16 & 3.78 \\
\hline \multirow[t]{2}{*}{ Glycyrrhizin } & 13.42 & 8.00 & 21.40 & 99.75 & 1.34 \\
\hline & & 16.00 & 30.07 & 104.05 & 1.14 \\
\hline
\end{tabular}

Conc., concentration; RSD, relative standard deviation $(\%)=(\mathrm{SD} /$ mean $) \times 100$.
Table 7. Reproducibility and stability of the 18 marker compounds (RSD, \%)

\begin{tabular}{lccc}
\hline \multirow{2}{*}{ Compound } & \multicolumn{2}{c}{ Reproducibility $(n=6)$} & \multirow{2}{*}{ Stability $(\leq 16 \mathrm{~h})$} \\
\cline { 2 - 3 } & Retention time & Absolute area & \\
\hline \hline Gallic acid & 0.04 & 0.79 & 1.10 \\
Oxypaeoniflorin & 0.06 & 0.75 & 0.85 \\
(+)-Catechin & 0.06 & 1.11 & 2.02 \\
Chlorogenic acid & 0.05 & 0.64 & 0.44 \\
Caffeic acid & 0.05 & 0.91 & 0.59 \\
Paeoniflorin & 0.06 & 1.06 & 0.42 \\
Ferulic acid & 0.06 & 0.95 & 0.58 \\
Liquiritin & 0.06 & 0.83 & 0.87 \\
Nodakenin & 0.07 & 0.97 & 0.55 \\
1,2,3,4,6-Penta & 0.08 & 1.64 & 3.28 \\
galloylglucose & & 1.18 & 0.18 \\
Coumarin & 0.06 & 0.88 & 0.58 \\
Ononin & 0.04 & 1.09 & 0.60 \\
Cinnamic acid & 0.04 & 1.09 & 0.85 \\
Cinnamaldehyde & 0.03 & 0.89 & 0.25 \\
Benzoylpaeoniflo & 0.02 & 0.86 & 0.50 \\
rin & 0.03 & 2.43 & 1.66 \\
Paeonol & 0.02 & 0.87 & 1.57 \\
6-Gingerol & 0.11 & & \\
Glycyrrhizin & &
\end{tabular}

$\mathrm{RSD}$, relative standard deviation $(\%)=($ standard deviation $/$ mean $) \times 100$.

\section{Quantification of the 18 marker compounds in OGT samples}

The validated method was successfully applied to determine and quantify the 18 marker compounds in OGTWE and two commercial OGT granules (OGT01 and OGT02)(Fig. 3).

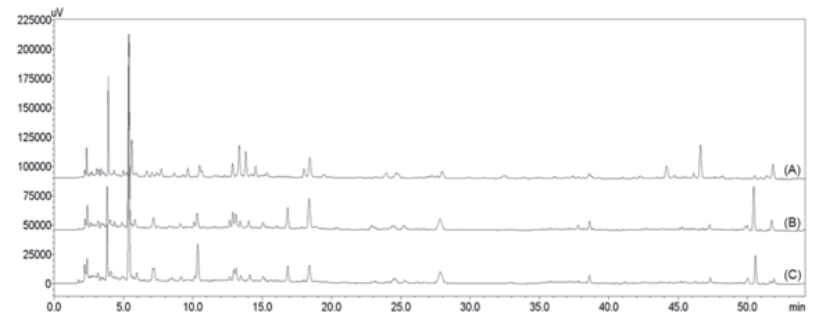

Fig. 3. Chromatograms of OGT water extract and OGT commercial granules at $250 \mathrm{~nm}$. (A) OGT water extract, (B) OGT01 (commercial OGT granules), and (C) OGT02 (commercial OGT granules).

In OGTWE, the amount of paeoniflorin was highest, followed by paeonol, while the amounts of 6-gingerol, $(+)$-catechin, ononin, and cinnamic acid were lowest. The amount of paeoniflorin was more than 500-fold compared to that of 6-gingerol. Furthermore, paeoniflorin and gallic acid were present in higher amounts, while ferulic acid and paeonol were present in lower amounts, in two commercial OGT granules in which (+)-catechin, caffeic acid, coumarin, cinnamaldehyde, and paeonol were not detected (and hence it was not possible to quantify these compounds). In addition, 1,2,3,4,6-pentagalloylglucose was not detected in the OGT01(Table 8). 
Table 8. Quantification of the 18 marker compounds in OGT samples

\begin{tabular}{lccc}
\hline \multirow{2}{*}{ Compound } & \multicolumn{3}{c}{ Content $(\mathrm{mg} / \mathrm{g})^{\mathrm{a}}$} \\
\cline { 2 - 4 } & OGTWE & OGT01 & OGT02 \\
\hline \hline Gallic acid & $0.985 \pm 0.007$ & $1.332 \pm 0.007$ & $1.649 \pm 0.005$ \\
Oxypaeoniflorin & $0.632 \pm 0.005$ & $0.095 \pm 0.004$ & $0.047 \pm 0.001$ \\
(+)-Catechin & $0.094 \pm 0.002$ & $\mathrm{ND}$ & $\mathrm{ND}$ \\
Chlorogenic acid & $0.987 \pm 0.001$ & $0.064 \pm 0.001$ & $0.054 \pm 0.000$ \\
Caffeic acid & $0.117 \pm 0.000$ & $\mathrm{ND}$ & $\mathrm{ND}$ \\
Paeoniflorin & $6.783 \pm 0.023$ & $2.818 \pm 0.043$ & $1.629 \pm 0.067$ \\
Ferulic acid & $0.295 \pm 0.001$ & $0.008 \pm 0.000$ & $0.006 \pm 0.000$ \\
Liquiritin & $1.250 \pm 0.006$ & $0.237 \pm 0.004$ & $0.281 \pm 0.002$ \\
Nodakenin & $1.599 \pm 0.010$ & $0.078 \pm 0.001$ & $0.106 \pm 0.002$ \\
1,2,3,4,6-Pentagalloylglucose & $0.191 \pm 0.004$ & $\mathrm{ND}$ & $0.016 \pm 0.000$ \\
Coumarin & $0.361 \pm 0.000$ & $\mathrm{ND}$ & $\mathrm{ND}$ \\
Ononin & $0.094 \pm 0.001$ & $0.042 \pm 0.000$ & $0.041 \pm 0.000$ \\
Cinnamic acid & $0.093 \pm 0.001$ & $0.012 \pm 0.000$ & $0.012 \pm 0.000$ \\
Cinnamaldehyde & $0.661 \pm 0.004$ & $\mathrm{ND}$ & $\mathrm{ND}$ \\
Benzoylpaeoniflorin & $0.275 \pm 0.001$ & $0.102 \pm 0.001$ & $0.046 \pm 0.000$ \\
Paeonol & $2.377 \pm 0.003$ & $0.006 \pm 0.000$ & $0.002 \pm 0.000$ \\
6-Gingerol & $0.013 \pm 0.000$ & $\mathrm{ND}$ & $\mathrm{ND}$ \\
Glycyrrhizin & $1.342 \pm 0.023$ & $0.195 \pm 0.002$ & $0.103 \pm 0.006$ \\
\hline
\end{tabular}

a Content is represented as 'average \pm standard deviation'.

ND, not detected; OGTWE, OGT water extract; OGT01 and OGT02, commercial granules from Korean manufacturers.

Variations in the amounts of the marker compounds were also found between samples. Gallic acid was the only compound that was present in a significantly higher amount in the commercial granules than in OGTWE, while the amounts of most detectable compounds in the commercial samples were significantly lower than those of OGTWE. The difference in the amounts of the marker compounds between OGT samples was about 2 -1200-fold(Fig. 4).
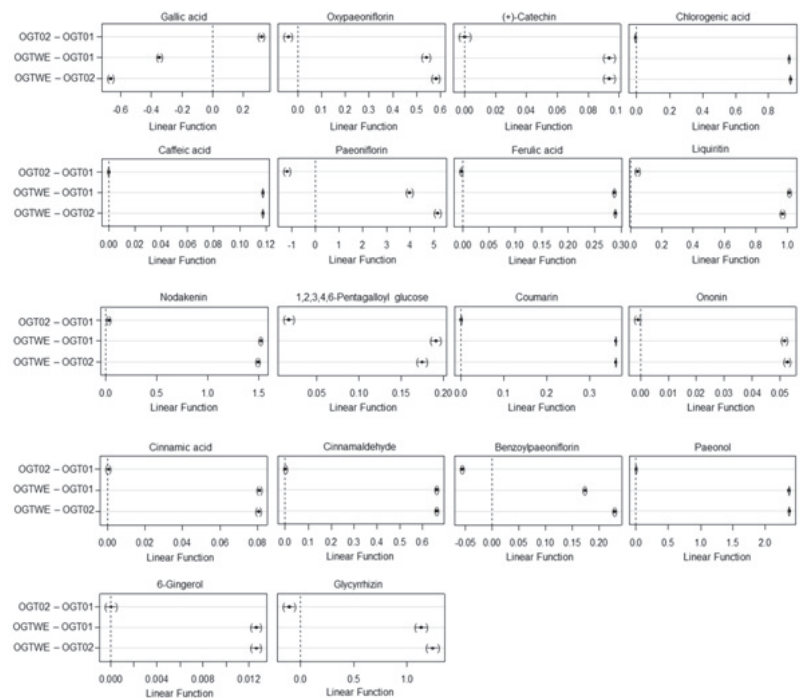

Fig. 4. One-way ANOVA using the Tukey test of the 18 marker compounds among samples with 95\% family-wise confidence level.

\section{Pearson correlation efficient and PCA of}

\section{OGT samples}

The relationship between OGT samples was represented by the Pearson correlation coefficient, using the quantification results. In the box plot, the median value of the coefficient of OGTWE was low compared with the values of the two commercial OGT granules(Fig. 5).

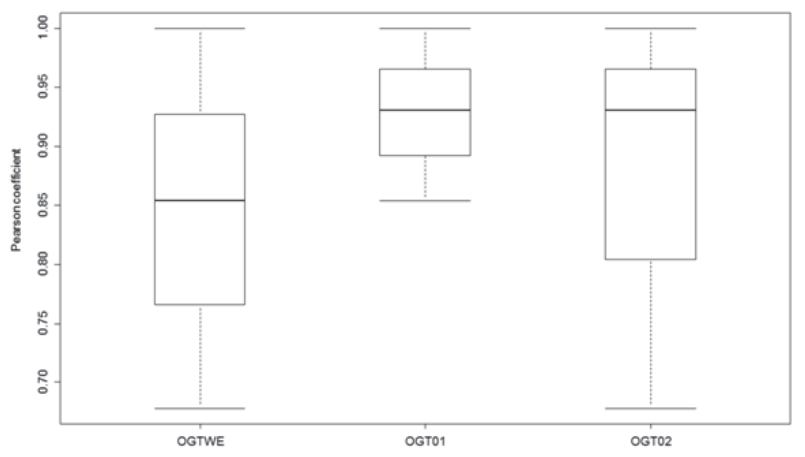

Fig. 5. Pearson coefficient of OGT samples. OGTWE, OGT water extract; OGT01-OGT02, commercial OGT granules

PCA results showed a distinct relationship between OGT samples. In the score plot, OGTWE was clearly separated from two commercial OGT samples by principal component 1 (PC1) with high total variance (98.3\%). Principal component 2 (PC2) score further divided the commercial granules into positive and negative scores, with an insignificant effect on total variance (1.7\%) (Fig. 6A). Of the 18 marker compounds on loading plot, only gallic acid was positioned at a positive PC1 score, while the 17 compounds were all positioned at a negative PC1 score(Fig. 6B).
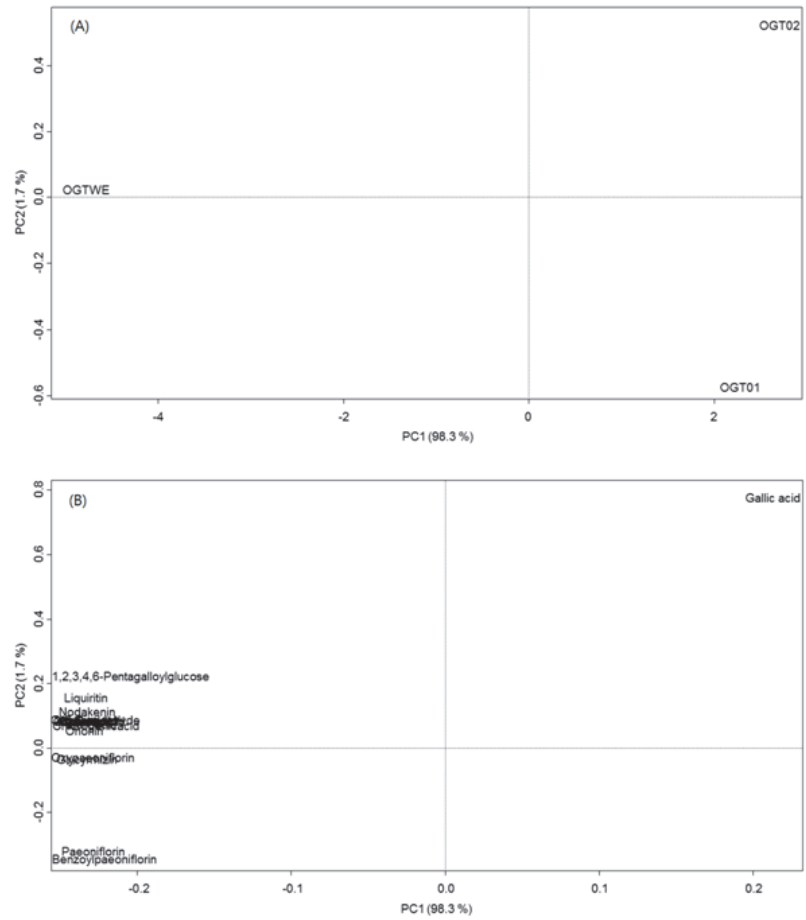

Fig. 6. Score plot (A) and loading plot (B) of principal components (PC1 vs. $\mathrm{PC2}$ ) on the variables (amounts of the 18 marker compounds) with the objectives (OGT samples). PC1 and PC2 contributed to $98.3 \%$ and $1.7 \%$ of total variance, respectively. OGTWE, OGT water extract; OGT01-OGT02, commercial OGT granules. 


\section{Discussion}

The HPLC conditions for the analysis of OGTWE were established; these included the mobile phase ratio, modifier, and UV wavelength of PDA. The reversed-phase $\mathrm{C}_{18}$ is the most frequently used stationary phase in herbal medicine analysis using a HPLC system ${ }^{28-30)}$. We, therefore, employed a $\mathrm{C}_{18}$ column for the simultaneous determination of the 18 marker compounds in OGTWE.

There were noticeable variation in the amounts of the 18 marker compounds observed between OGTWE and two commercial granules. Of the marker compounds of which amounts were significantly higher in OGTWE, the amount of gallic acid was solely higher in the commercial granules than in OGTWE. This is because cinnamaldehyde and paeonol are volatile; therefore, so they can be easily vaporized at high temperature, without causing the vapour valve of the extractor to be closed and $(+)$-catechin is degraded at high temperature ${ }^{31-33)}$. The other compounds, except for gallic acid, were not extracted efficiently because of the possible interference from other components, or an excessive amount of excipient.

The result from Pearson correlation coefficient showed that the median value of the coefficient of OGTWE was apparently different from those of the two commercial OGT granules. This result indicates that OGTWE was not closely correlated with the two commercial samples. The principal component analysis (PCA) result also supported the result of Pearson correlation coefficient. In the score plot, principal component 1 (PC1), which is most influential to sample clustering, significantly contributed to separation of OGTWE from two commercial OGT samples. Principal component 2 (PC2) score, next influential variable of sample distribution orthogonal to $\mathrm{PC1}$, also divided two commercial granules with $1.7 \%$ of total variance, although influence was not significant. This means that the two commercial samples are closely related, while OGTWE is not associated with those samples-closer PC scores means a closer relationship ${ }^{34)}$. In loading plot, only gallic acid was conglomerated far from 17 compounds, which indicates that the compounds located at each PC score contributed to the classification of OGT samples and gallic acid is most potential compound for separation of OGTWE and commercial OGT samples.

Taken together, the results from the quantification of 18 marker compounds, Pearson correlation coefficient, and PCA, the commercial OGT samples were significantly different from laboratory-produced OGT water extract. Moreover, there is variations in the amounts of several compounds within commercial OGT samples. These are presumably ascribed to the difference of manufacturing process among companies or herbal resources used, which led to variations in the amounts of marker compounds. It is the reason why a similar processing method of herbal preparation among the companies is much needed to maintain constant quality of OGT preparations.

\section{Conclusions}

In the present study, the analytical methods of HPLC-PDA, using reversed-phase $\mathrm{C}_{18}$ column, was developed for the simultaneous determination of the 18 marker compounds in OGTWE.

1. Validation methods included system suitability, precision, recovery, reproducibility, and stability. The developed method was precise, reproducible and reliable, and successfully applied to the quantification of OGTWE and commercial OGT granules.

2. Variation in the amounts of the 18 marker compounds were observed among OGT samples.

3. The relationship between OGTWE and commercial granules, as evaluated by the Pearson correlation coefficient and PCA, was not close.

The results of this analytical study provide chemical information on OGT preparations. The quantitative analysis developed will be useful for further quality assessment.

\section{Conflict of interest}

The authors declare no conflict of interest in this work

\section{Acknowledgements}

This study was supported by a grant from the Korea Institute of Oriental Medicine (no. K14030)

\section{References}

1. The Korean Medicine Society for the Herbal Formula Study. Herbal formula. Younglimsa : Seoul. 2003 : 420.

2. Ushiroyama T, Tsubokura S, Ikeda A, Ueki M. The 
Effect of Unkei-to on pituitary gonadotropin secretion and ovulation in anovulatory cycles of young women. Am J Chin Med. 1995 ; 23 : 223-30.

3. Ushiroyama T, Ikeda A, Sakai M, Hosotani T, Suzuki Y, Tsubokura S, Ueki M. Effects of unkei-to, an herbal medicine, on endocrine function and ovulation in women with high basal levels of luteinizing hormone secretion. J Reprod Med. 2001 ; 46 : 451-6.

4. Ushiroyama T, Hosotani T, Yamashita Y, Yamashita H, Ueki M. Effects of Unkei-to on FSH, LH and estradiol in anovulatory young women with hyperor hypo-functioning conditions. Am J Chin Med. 2003 ; 31 : 763-71.

5. Koike K, Ohno S, Takahashi N, Suzuki N, Nozaki N, Murakami K, Sugiura K, Yamada K, Inoue M. Efficacy of the herbal medicine Unkei-to as an adjunctive treatment to hormone replacement therapy for postmenopausal women with depressive symptoms. Clin Neuropharmacol. 2004 ; 27 : 157-62.

6. Yasui T, Matsuzaki T, Ogata R, Kiyokawa M, Ushigoe K, Uemura H, Kuwahara A, Ikawa H, Maegawa M, Furumoto H, Aono T, Irahara M. The herbal medicine Unkei-to stimulates the secretion of a cytokine-induced neutrophil chemoattractant, CINC/gro, in the rat ovarian cell culture. Am J Reprod Immunol. 2003 ; 49 : 14-20.

7. Sun WS, Imai A, Tagami K, Sugiyama M, Furui T, Tamaya $\mathrm{T}$. In vitro Stimulation of granulosa cells by a combination of different active ingredients of Unkei-to. Am J Chin Med. 2004 ; 32 : 569-78.

8. Terawaki K, Koike K, Yuzurihara M, Kase Y, Takeda S, Aburada M, Murakami K, Ohno S, Suzuki N, Inoue M. Effects of the traditional Japanese medicine Unkei-to on the corticotropin-releasing factor-induced increase in locomotor activity. Pharmacol Biochem Behav. 2004 ; $78: 799-803$

9. Kim HI, Lim EM, Kwon KR. Effects of Onkyung-tang on surgically induced endometriosis in rats. $J$ Orient Obstet Gynecol. 2006 ; 19 : 18-33.

10. Li H, Wang SW, Zhang BL, Xie YH, Yang Q, Cao W, Wang JB. Simultaneous quantitative determination of 9 active components in traditional Chinese medicinal preparation ShuangDan oral liquid by RP-HPLC coupled with photodiode array detection. J Pharm Biomed Anal. 2011 ; 56 : 820-4.

11. Tang Y, Zhang Y, Li W, Guo S, Zhu M, Su S, Zhang L, Ding A, Duan JA. Comparative characterization of ten aromatic acids in Siwu series decoctions and their constituting herbs by HPLC-DAD method. J Liq Chromatogr Relat Technol. $2012 ; 35$ : 2425-38.

12. Su S, Cui W, Zhou W, Duan JA, Shang E, Tang Y. Chemical fingerprinting and quantitative constituent analysis of Siwu decoction categorized formulae by UPLC-QTOF/MS/MS and HPLC-DAD. Chin Med. $2013 ; 8: 5$.

13. Wang $X$, Lu H, Sun $H$, Jiang $X, W u ~ Z$, Sun $W$, Wang P, Liu L, Bi K. Quality evaluation of Yin Chen Hao Tang extract based on fingerprint chromatogram and simultaneous determination of five bioactive constituents. J Sep Sci. 2008 ; 31 : 9-15.

14. $\mathrm{Hu} \mathrm{Y}, \mathrm{Lu} \mathrm{T}$, Mao C, Wu H, Zhang X, Wang JV, Gu $J$. Simultaneous determination of 10 components in traditional Chinese medicine Dachaihu granule by reversed -phase-high-performance liquid chromatographic - diode array detector. Phcog Mag. $2013 ; 9$ : 33-8.

15. Wang B, Shen L, Cong W, Lin X, Feng Y, Zhu Y, Wang Q. A simple HPLC method for simultaneous analysis of 7 bioactive constituents for Liuwei Dihuang Pill and its application in quality consistency evaluation. Anal Methods. 2013 ; 5 : 2384-90.

16. Chen WJ, Chang CY, Lin JK. Induction of G1 phase arrest in MCF human breast cancer cells by pentagalloylglucose through the down-regulation of CDK4 and CDK2 activities and up-regulation of the CDK inhibitors $\mathrm{p} 27^{\mathrm{Kip}}$ and $\mathrm{p} 21^{\mathrm{Cip}}$. Biochem Pharmacol. 2003 ; 65 : 1777-85.

17. Lee SC, Kwon YS, Son KH, Kim HP, Heo MY. Antioxidative constituents from Paeonia lactiflora. Arch Pharm Res. 2005 ; 28 : 775-83.

18. Tak JH, Kim HK, Lee SH, Ahn YJ. Acaricidal activities of paeonol and benzoic acid from Paeonia suffruticosa root bark and monoterpenoids against Tyrophagus putrescentiae (Acari : Acaridae). Pest Manag Sci. 2006 ; 62 : 551-7.

19. Koo YK, Kim JM, Koo JY, Kang SS, Bae KH, Kim YS, Chung JH, Yun-Choi HS. Platelet anti-aggregatory and blood anti-coagulant effects of compounds isolated from Paeonia lactiflora and Paeonia suffruticosa. Die Pharmazie. 2010 ; 65 : 624-8.

20. Li W, Tang Y, Chen Y, Duan JA. Advances in the chemical analysis and biological activities of chuanxiong. Molecules. 2012 ; 17 : 10614-51.

21. Ryu YB, Kim JH, Park SJ, Chang JS, Rho MC, Bae KH, Park KH, Lee WS. Inhibition of neuraminidase activity by polyphenol compounds isolated from the roots of Glycyrrhiza uralensis. Bioorg Med Chem Lett. 2010 ; 20 : 971-4.

22. Rim HK, Cho W, Sung SH, Lee KT. Nodakenin suppresses lipopolysaccharide-induced inflammatory responses in macrophage cells by inhibiting tumor necrosis factor receptor-associated factor 6 and 
nuclear factor $-\kappa \mathrm{B}$ pathways and protects mice from lethal endotoxin shock. J Pharmacol Exp Ther. 2012 ; 342 : 654-64.

23. Kim SY, Koo YK, Koo JY, Ngoc TM, Kang SS, Bae KH, Kim YS, Yun-Choi HS. Platelet Anti-aggregation activities of compounds from Cinnamomum cassia. J Med Food. 2010 ; 13 : 1069-74.

24. Liao JC, Deng JS, Chiu CS, Hou WC, Huang SS, Shie PH, Huang GJ. Anti-Inflammatory activities of Cinnamomumcassia constituents in vitro and in vivo. Evid Based Complement Alternat Med. 2012 ; 2012 : 429320.

25. Dugasani S, Pichika MR, Nadarajah VD, Balijepalli MK, Tandra S, Korlakunta JN. Comparative antioxidant and anti-inflammatory effects of [6]-gingerol, [8]-gingerol, [10]-gingerol and [6]-shogaol. J Ethnopharmacol. $2010 ; 127$ : 515-20.

26. Yang J, Chen LH, Zhang Q, Lai MX, Wang Q. Quality assessment of Cortex cinnamomi by HPLC chemical fingerprint, principle component analysis and cluster analysis. J Sep Sci. 2007 ; 30 : 1276 -83 .

27. Xu S, Yang L, Tian R, Wang Z, Liu Z, Xie P, Feng Q. Species differentiation and quality assessment of Radix Paeoniae Rubra (Chi-shao) by means of high-performance liquid chromatographic fingerprint. J Chromatogr A. 2009 ; 1216 : 2163-8.

28. Borrás Linares I, Arráez-Román D, Herrero M, Ibáñez E, Segura-Carretero A, Fernández-Gutiérrez A. Comparison of different extraction procedures for the comprehensive characterization of bioactive phenolic compounds in Rosmarinus officinalis by reversed-phase high-performance liquid chromatography with diode array detection coupled to electrospray time-of-flight mass spectrometry. J Chromatogr A. 2011 ; 1218 : 7682-90.

29. He D, Shan Y, Wu Y, Liu G, Chen B, Yao S. Simultaneous determination of flavanones, hydroxycinnamic acids and alkaloids in citrus fruits by HPLC-DAD-ESI/MS. Food Chem. 2011 ; $127: 880-5$.

30. Niu TZ, Zhang YW, Bao YL, Wu Y, Yu CL, Sun LG, Yi JW, Huang YX, Li YX. A validated high-performance liquid chromatography method with diode array detection for simultaneous determination of nine flavonoids in Senecio cannabifolius Less. J Pharm Biomed Anal. 2013 ; 76 : 44-8.

31. Shim SL, Hwang IM, Ryu KY, Jung MS, Seo HY, Kim HY, Song HP, Kim JH, Lee JW, Byun MW, Kwon JH, Kim KS. Effect of $\gamma$-irradiation on the volatile compounds of medicinal herb, Paeoniae Radix. Radiat Phys Chem. 2009 ; 78 : 665-9.

32. Wang R, Wang R, Yang B. Extraction of essential oils from five cinnamon leaves and identification of their volatile compound compositions. Innov Food Sci Emerg Technol. 2009 ; 10 : 289-92.

33. Perva-Uzunalić A, Škerget M, Knez Ž, Weinreich B, Otto F, Grüner S. Extraction of active ingredients from green tea (Camellia sinensis): Extraction efficiency of major catechins and caffeine. Food Chem. 2006 ; 96 : 597-605.

34. Lu J, Wang JS, Kong LY. Anti-inflammatory effects of Huang-Lian-Jie-Du decoction, its two fractions and four typical compounds. J Ethnopharmacol. $2011 ; 134$ : 911-8. 